\title{
Antenna Design for Directivity-Enhanced Raman Spectroscopy
}

\author{
Aftab Ahmed, Yuanjie Pang, Ghazal Hajisalem, and Reuven Gordon \\ Department of Electrical and Computer Engineering, University of Victoria, Victoria, BC, Canada V8W 3P6 \\ Correspondence should be addressed to Reuven Gordon, rgordon@uvic.ca
}

Received 1 October 2011; Revised 22 December 2011; Accepted 4 January 2012

Academic Editor: Nicolas Bonod

Copyright () 2012 Aftab Ahmed et al. This is an open access article distributed under the Creative Commons Attribution License, which permits unrestricted use, distribution, and reproduction in any medium, provided the original work is properly cited.

\begin{abstract}
Antenna performance can be described by two fundamental parameters: directivity and radiation efficiency. Here, we demonstrate nanoantenna designs in terms of improved directivity. Performance of the antennas is demonstrated in Raman scattering experiments. The radiated beam is directed out of the plane by using a ground plane reflector for easy integration with commercial microscopes. Parasitic elements and parabolic and waveguide nanoantennas with a ground plane are explored. The nanoantennas were fabricated by a series of electron beam evaporation steps and focused ion beam milling. As we have shown previously, the circular waveguide nanoantenna boosts the measured Raman signal by $5.5 \mathrm{x}$ with respect to a dipole antenna over a ground plane; here, we present the design process that led to the development of that circular waveguide nanoantenna. This work also shows that the parabolic nanoantenna produces a further fourfold improvement in the measured Raman signal with respect to a circular waveguide nanoantenna. The present designs are nearly optimal in the sense that almost all the beam power is coupled into the numerical aperture of the microscope. These designs can find applications in microscopy, spectroscopy, light-emitting devices, photovoltaics, single-photon sources, and sensing.
\end{abstract}

\section{Introduction}

Antennas have been widely used in radio communications for more than a century for efficient transmission of information over long distances. Since its discovery in 1895, enormous progress has been made with better control of antenna parameters (for a brief history see [1] and references therein). For example, directional emission was demonstrated by YagiUda in the microwave regime [2] where the radiation from the feed element is directed with the assistance of reflector and director parasitic elements. The directivity of antennas has played a vital role in microwave communication systems, specifically in satellite communication for the realization of high-gain antennas.

Radio antennas provide solutions to communication problems, whereas recent developments for realization of optical antennas were mainly dictated by microscopy and spectroscopy applications $[3,4]$. Typically antennas have dimensions of the order of the operating wavelength, requiring antenna dimensions in nanometers for operation in the visible regime. In the visible-IR regime, the metal cannot be taken as a perfect conductor and the nanoantenna design must be modified due to plasmonic properties [5-8]. Recent developments in the nanotechnology have made the fabrication of such small structures possible, leading to the development of optical single element and Yagi-Uda equivalent nanoantennas [9-20]. Directing the emission from optical emitters is highly desired for efficient detection and, by reciprocity, efficient excitation as well. Typical applications include light-emitting devices [21,22], photovoltaics [2327], sensing [28, 29], spectroscopy [30-32], single-photon sources $[33,34]$, and microscopy $[3,35,36]$.

In a recent work, our group has shown that the scattered radiation from nanoparticles can be directed out of the plane of substrate and into the collection microscope using a ground plane reflector [37]. Enhancement of over 50x was observed in the measured Raman signal as compared to nanoprisms over a glass substrate. We also demonstrated recently an experimental work on waveguide nanoantenna to boost the Raman signal by beam forming in the lateral plane [38]. A further 5.5x enhancement was observed with a circular waveguide nanoantenna as compared to a dipole nanoantenna over a ground plane.

The main objective of this work is to present the design of planar nanoantennas for optimal performance in directivityenhanced Raman scattering (DERS). Here, we present the 
details of the design process that led to the development of circular waveguide nanoantenna, the different designs considered for beam forming in the lateral plane, and the possibility of higher local fields for further Raman enhancement. We also show experimentally that the parabolic reflector can enhance the Raman signal by $4 \mathrm{x}$ as compared to the circular waveguide nanoantenna owing to its improved directivity and enhanced local fields; however, it is challenging to fabricate.

\section{Antenna Design Parameters}

The total power radiated by an antenna can be given as

$$
P_{\mathrm{rad}}=\int_{0}^{2 \pi} \int_{0}^{\pi} p(\theta, \phi) \sin (\theta) d \theta d \phi
$$

where $p(\theta, \phi)$ is the normalized angular power density also known as radiation pattern of an antenna. Directivity is defined as the antennas ability to radiate in a specific direction more efficiently as compared to a hypothetical isotropic radiator [39]:

$$
D=\frac{4 \pi}{P_{\mathrm{rad}}} p(\theta, \phi)
$$

Considering the numerical aperture (NA) of the microscope objective, a more suitable parameter for describing the beam forming ability of an antenna is the beam efficiency (BE):

$$
\mathrm{BE}=\frac{\int_{0}^{2 \pi} \int_{0}^{\theta_{0}} p(\theta, \phi) \sin (\theta) d \theta d \phi}{P_{\mathrm{rad}}}
$$

where $\theta_{0}$ is the cone half angle. Cone angle of 30 degrees was calculated from the measured spot size " $d$ " of approximately $1.5 \mu \mathrm{m}$, using $\theta_{0}=\sin ^{-1}(\lambda / 2 d)$. This corresponds to an NA of 0.28 .

Radiation patterns of single element nanoantennas are typically dipolar in nature resulting in poor directivity $\left(D_{\max }=1.5\right)$. Directive emission at optical wavelengths has been achieved using multielement nanoantennas $[11,13,40]$. Radiation patterns of those antennas reveal that the main beam is directed in the plane of the substrate, thus cannot be readily used in an ordinary microscope setup. Further, vertical Yagi-Uda nanoantenna has been realized using top-down approach [15]. Improved directivity at optical wavelengths can have tremendous impact in areas such as optical microscopy, spectroscopy, sensing, and applications involving single-photon sources, where efficient collection and emission is critical.

Another important factor in the context of Raman measurements is the local field strength. It has been recently shown that maximum field enhancement results when power radiated by the antenna is equal to the power loss in the antenna [41-43]. This is commonly referred to as impedance matching in microwave antenna theory [39]. Even higher local field can be achieved by reducing the mode volume of the antenna by reducing the feed gap between the two elements of the dipole antenna [43].

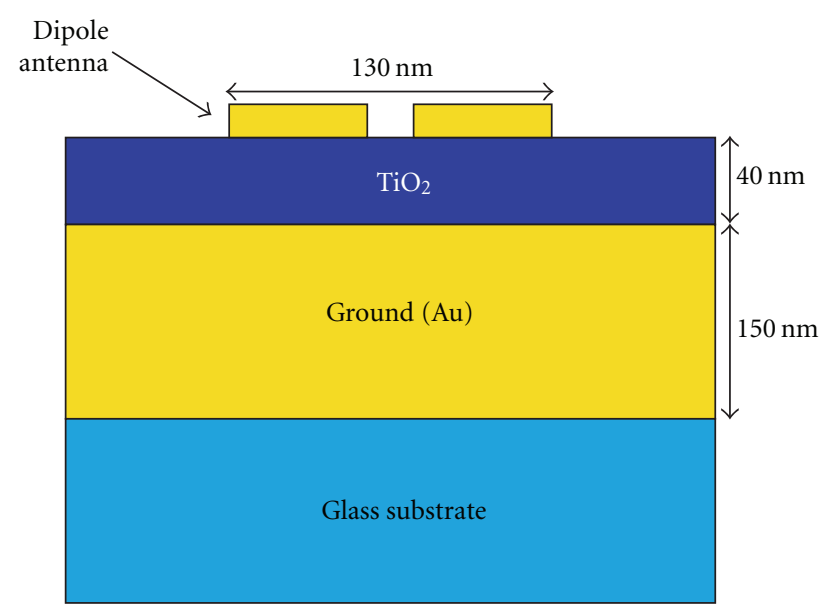

FIGURE 1: Multilayer substrate to control the radiation pattern of a dipole antenna. Thicknesses of ground plane, dielectric $\left(\mathrm{TiO}_{2}\right)$ spacer, and top gold layer are $150 \mathrm{~nm}, 40 \mathrm{~nm}$, and $50 \mathrm{~nm}$, respectively. Dipole antenna length of $130 \mathrm{~nm}$ with a $20 \mathrm{~nm}$ feed gap was used to operate at wavelength of $840 \mathrm{~nm}$.

\section{Design and Fabrication}

In this paper we investigate different nanoantenna designs for DERS with the desired features of out-of-plane radiation and enhanced local fields. The introduction of ground plane prevents loss of scattered radiation into the substrate and proper adjustment of antenna distance from the ground plane can result in radiation enhancement out of the plane. Further improvement of directivity is demonstrated by beam shaping in the lateral plane using different designs including parasitic elements, waveguide antennas, and parabolic reflectors. The multilayer substrate is fabricated by electron beam evaporation, and the different designs are milled using focused ion beam (FIB).

Figure 1 illustrates the multilayer substrate used for the fabrication of nanoantennas studied in this work. The antennas were designed to be tested under Raman microscope. The dimensions of different layers and the dipole antenna were calculated to achieve best performance at wavelength of $840 \mathrm{~nm}$ (mean of excitation and Raman scattered wavelengths). The antenna consists of a $130 \mathrm{~nm}$ long and $50 \mathrm{~nm}$ wide dipole. Traditionally, dipole antenna characteristic lengths were of the order of wavelength of operation $(\lambda / 2)$, but the real metal response requires that shorter effective wavelengths are introduced for the determination of dipole length in infrared and optical regions [7]. A $150 \mathrm{~nm}$ thick gold layer was used as ground plane to ensure that it was optically thick.

The optimal thickness of dielectric spacer $\left(\mathrm{TiO}_{2}\right)$ was calculated numerically to be $40 \mathrm{~nm}$ using finite-difference time-domain (FDTD) simulations. It should be noted that this is smaller than the typical quarter wavelength value due to penetration into the metal, as well as impedance matching effects [43].

The multilayer substrate was fabricated by evaporation of gold and $\mathrm{TiO}_{2}$ onto glass substrate by means of electron beam evaporation under a pressure of $2 \times 10^{-6}$ Torr. The 


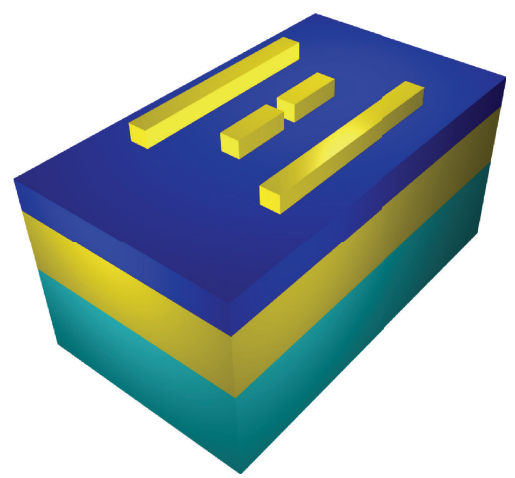

(a)

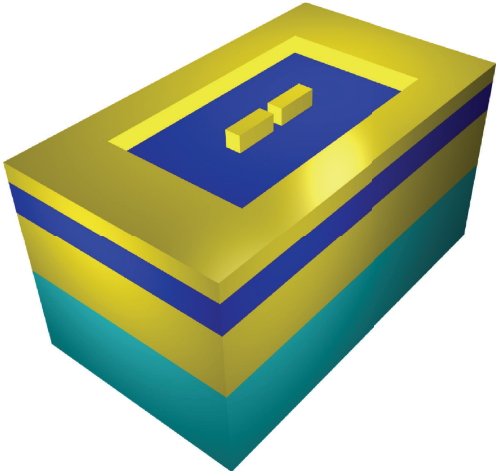

(b)

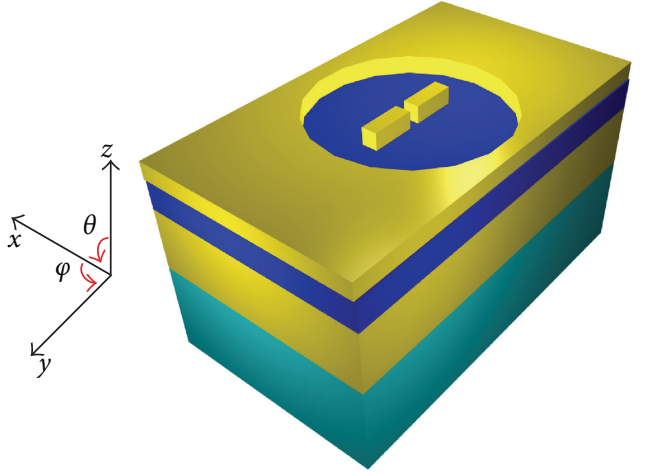

(c)

FIGURE 2: (a) Dipole antenna over ground with two parasitic reflectors $150 \mathrm{~nm}$ long and $100 \mathrm{~nm}$ from the feed element. (b) Dipole antenna over ground plane with a square reflector of length $500 \mathrm{~nm}$ (square waveguide antenna). (c) Dipole antenna over ground plane with a circular reflector of radius $250 \mathrm{~nm}$ (circular waveguide antenna).

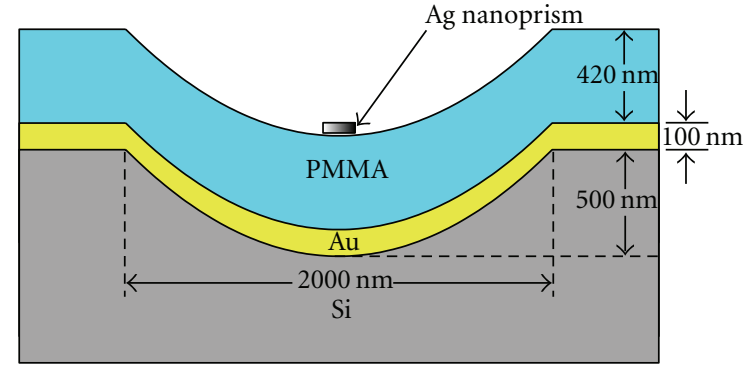

FIGURE 3: The schematic drawing of the proposed parabolic reflector nanoantenna.

proposed nanoantenna structures were milled on the top $50 \mathrm{~nm}$ thick gold layer using FIB. Figure 2 shows the different nanoantennas investigated in this work for normal emission. Relative efficiencies of these structures are discussed in the simulation section below, where it is shown that the circular waveguide nanoantenna shown in Figure 2(c) results in best performance in terms of directivity.

Another promising design translated from the microwave antenna theory is that of the parabolic reflector antenna. We demonstrate DERS from the beam forming abilities of a parabolic reflector nanoantenna. Figure 3 shows the schematic of the parabolic nanoantenna. A paraboloidshaped trench was milled into a silicon wafer using FIB. As shown in the figure, the focal length of this paraboloid was designed to be $500 \mathrm{~nm}$ and the diameter of the top circle is $2 \mu \mathrm{m}$. A layer of $100 \mathrm{~nm}$ thick gold (optically thick) was evaporated onto the silicon wafer by means of electron beam evaporation under a pressure of $2 \times 10^{-6}$ Torr.

The conformity of the evaporated layer to the hole in silicon creates a parabolic reflector antenna on Au layer. PMMA was then spin-coated on top of the Au layer as a spacing dielectric layer. We chose PMMA and the spincoating technique to make the spacer layer for reasons of simplicity and repeatable thickness control. Finally, Ag nanoprisms, synthesized in water by white-light assisted conversion of spherical nanoparticles [44], were mixed with
$3 \mu \mathrm{Mol}$ rhodamine $6 \mathrm{G}$ dye and drop-coated onto the PMMA surface. The nanoprisms serve as the feed of the parabolic reflector nanoantenna.

The experiments of [37] are first repeated using PMMA for the determination of optimal thickness of the PMMA layer. As a result (not shown), the first- and second-order coherent SERS enhancement peaks are found at $120 \mathrm{~nm}$ and $420 \mathrm{~nm}$ PMMA thickness. We choose to spin-coat $420 \mathrm{~nm}$ PMMA (corresponding to the second-order SERS enhancement) onto our parabolic reflector since this thickness is more compatible with the focal length of our parabolic reflector, bringing the feed Ag nanoprism near the paraboloid focal point.

\section{Simulation Results}

The proposed structures were simulated using the FDTD method. The simulation domain was terminated by perfectly matched layers (PMLs) for minimal reflections. The antenna structure was enclosed by a set of $2 \mathrm{D}$ field monitors forming a box to perform far-field projections and for the determination of field patterns. For the antenna structures shown in Figure 2, an electric dipole source located at the feed gap of the dipole antenna was used to excite the nanoantenna.

FDTD simulations indicate the following parameters for best performance for each type of antenna. Two $150 \mathrm{~nm}$ long parasitic reflectors, $100 \mathrm{~nm}$ away from the feed element, resulted in a half power beam width (HPBW) of 110 degrees in the $x z$ plane. Radiation patterns of this structure show a very broad HPBW in the $y z$ plane. To further improve the directivity in both planes we introduced reflectors parallel to the $x$-axis as well. It was demonstrated numerically that a square reflector with length of $500 \mathrm{~nm}$ resulted in best performance. The corresponding HPBWs are 85 degrees and 90 degrees in the $x z$ and $y z$ planes, respectively. Even better performance is observed by using a circular waveguide antenna as shown in Figure 2(c), producing a symmetrical beam out of the plane of substrate with HPBW of 85 degrees in both planes. Figures $4(\mathrm{a})$ and $4(\mathrm{~b})$ shows the radiation 


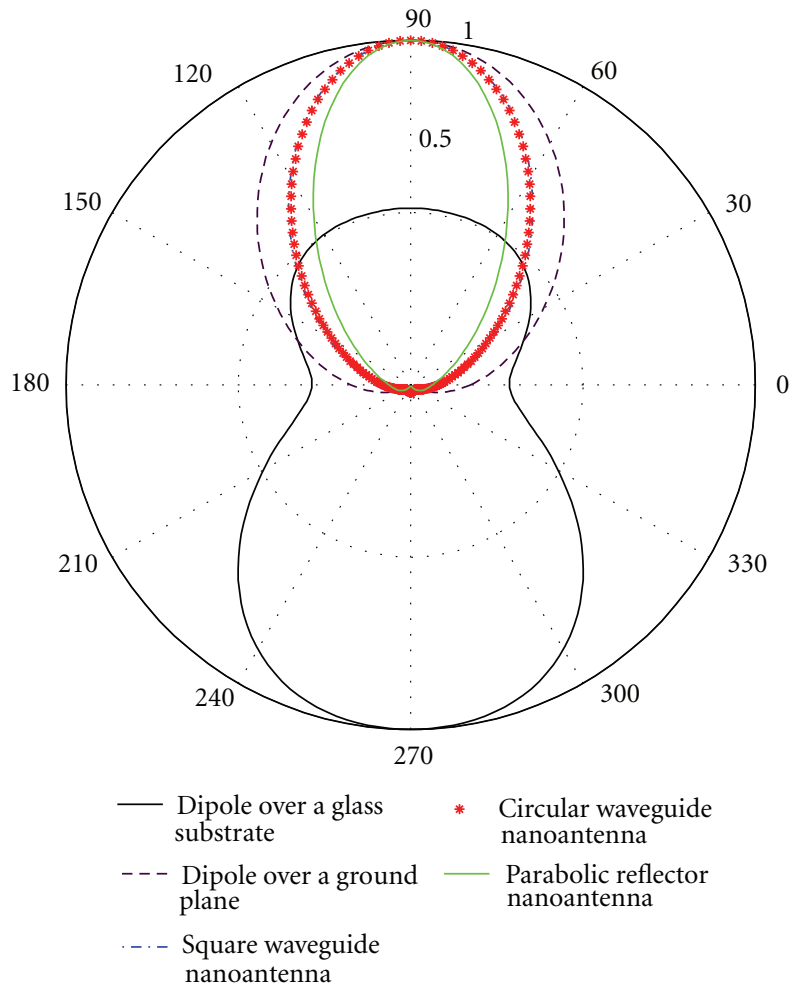

(a)

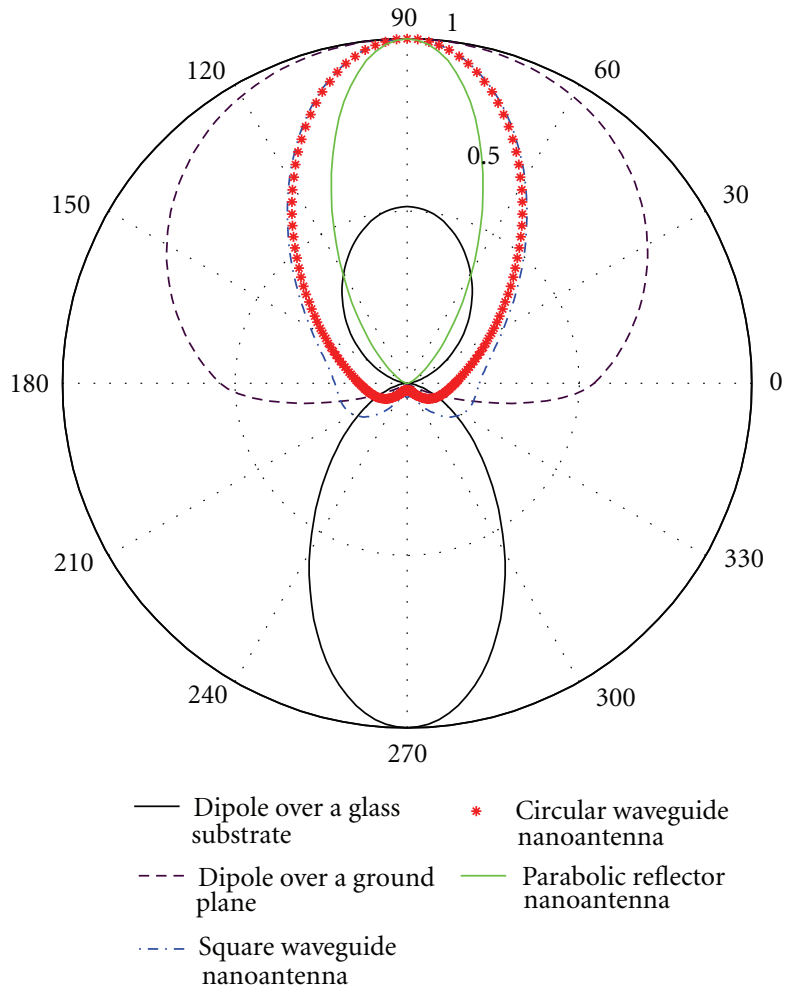

(b)

FIGURE 4: Radiation patterns of a dipole antenna on a glass substrate, dipole over ground plane, square waveguide nanoantenna $($ side length = $500 \mathrm{~nm}$ ), circular waveguide nanoantenna (radius $=250 \mathrm{~nm}$ ), and parabolic reflector nanoantenna. Calculated using far-field projections of 3D FDTD simulations, (a) $x z$ plane, (b) $y z$ plane.

pattern in the $x z$ and $y z$ planes, respectively, at the wavelength of $840 \mathrm{~nm}$. It can be seen that in the absence of ground reflector, most of the scattered power is directed into the substrate and away from the microscope objective.

The circular waveguide nanoantenna gives a nearly optimal radiation pattern resulting in the collection of almost all of the scattered light by a numerical aperture of 0.75 . The radiation patterns of the parabolic reflector are also plotted in Figure 4 for comparison. It can be seen that the parabolic reflector nanoantenna results in an even better directivity as compared to the circular waveguide nanoantenna.

The ring reflector acts to create a lateral standing wave that reflects light back towards the central dipole antenna structure. This is most similar to the waveguide antenna, which has a lateral resonance when the wavelength is $3.4 \mathrm{x}$ the radius of the circular waveguide (i.e., at the lowest order mode cut-off) $[39,45]$. Larger and smaller radii do not provide this resonance at the desired wavelength of $840 \mathrm{~nm}$ and thus give smaller directivities. The radiation patterns of circular waveguide nanoantenna with slightly smaller and larger radii (not shown) show splitting of the main beam into two lobes, thus lowering the directivity in the normal direction.

Since the present design is optimized to work at around $840 \mathrm{~nm}$, this corresponds to a radius of $250 \mathrm{~nm}$, which is precisely the radius value that was found to give the greatest DERS in the experiment. The beam forming for this antenna design allows for directive emission into a numerical aperture of approximately 0.75 ; therefore, it is well suited for microscope setups.

Another parameter dictating the intensity of Raman signal is the local field enhancement that arises from plasmonic resonances, tapers, gaps, and high curvature in the antenna design $[46,47]$. Impedance matching and reduction of mode volume of the antenna provide maximum local field enhancement [43]. Figures 5(a) and 5(b) show the local field intensity in the antenna gap for gap sizes of 20 and $5 \mathrm{~nm}$, respectively, at the design wavelength of $840 \mathrm{~nm}$ (log scale).

By reducing the gap size from $20 \mathrm{~nm}$ to $5 \mathrm{~nm}$, the normalized $E$ field intensity $\left(|E| /\left|E_{0}\right|\right)^{2}$ increases by approximately 1000x. Intensity of Raman signal is proportional to the square of $E$ field intensity, thus we expect an enhancement of $10^{6}$. The feed gap of the fabricated circular waveguide nanoantenna was $20 \mathrm{~nm}$. Thus, it is clear that the Raman signal can be considerably enhanced by reducing the feed gap, which is a challenging task.

Now we present the simulation results of the parabolic reflector nanoantenna. Figure 6 shows the electric field intensity profile at a vertical segment of the structures at the design wavelength of $840 \mathrm{~nm}$. It can be seen that the local field intensity at the Ag prism over a parabolic reflector is much larger than that over a planar reflector. From the 


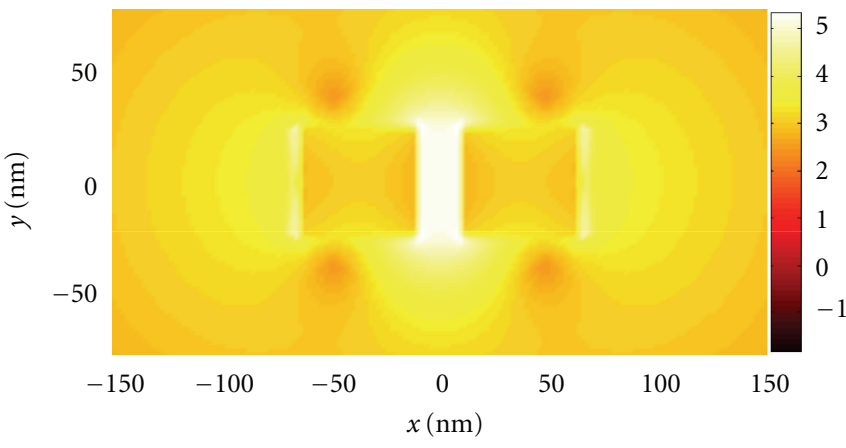

(a)

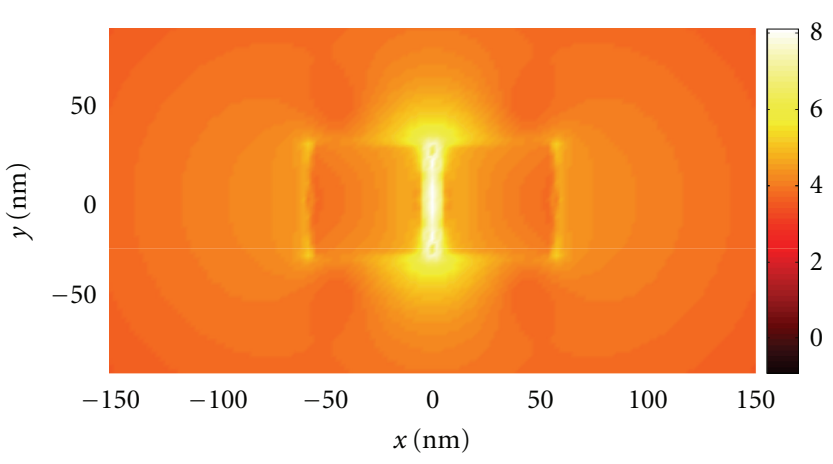

(b)

Figure 5: $E$ field intensity $\left(|E| /\left|E_{0}\right|\right)^{2}$ at the design wavelength of $840 \mathrm{~nm}$ for (a) gap size of $20 \mathrm{~nm}$ and (b) gap size of $5 \mathrm{~nm}$ (log scale).
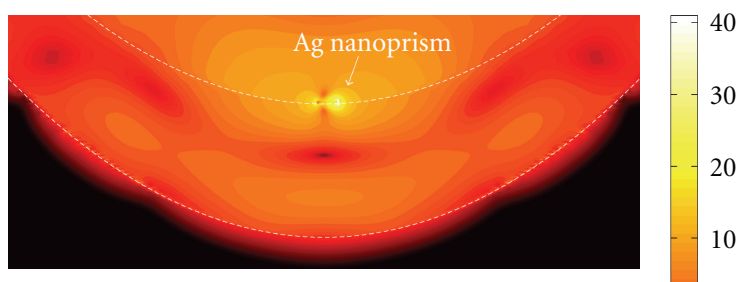

(a)

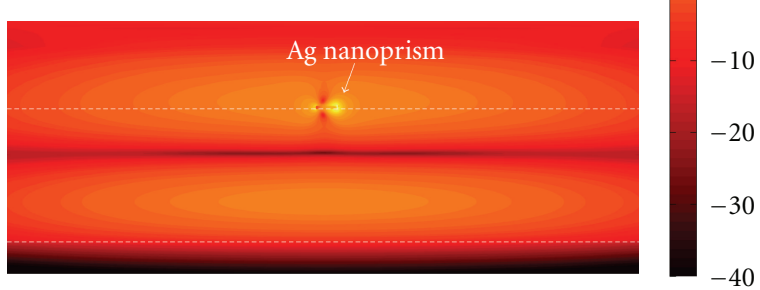

(b)

FIGURE 6: FDTD simulation results comparing the vertical segment electric field intensity profiles of an Ag nanoprism above (a) a parabolic reflector and (b) a planar reflector. The white dotted lines show the Au ground plane and the PMMA layer surfaces. The color map is in $\mathrm{dB}$ scale.

field intensity profiles in the PMMA layer, it can be seen that the Ag nanoprism is placed at the second antinode of the parabolic reflector at $840 \mathrm{~nm}$ wavelength, the same as in the planar reflector case; it is also at the focal point of the paraboloid. Therefore, the two effects, constructive interference and focusing, combine to give the enhanced Raman signal. Our parabolic reflector is able to collect more power from the incoming Gaussian wave to the Ag nanoprism feed element, indicating a better coupling between the far and the near field or, in a conventional antenna concept, a higher directivity.

In terms of Raman enhancement at the Ag nanoprism feed element, the relative Raman signal enhancement is proportional to the product of the excitation field intensity $\left|E_{\text {exc }}\right|^{2}$ and the emitted Raman field intensity $\left|E_{\text {raman }}\right|^{2}$. The Raman enhancement factor was computed at the near field of the Ag prism (within a rectangular box $10 \mathrm{~nm}$ away from the Ag prism). It was found from the local field enhancement that the Raman enhancement near an Ag prism in a parabolic reflector arrangement is $40 \mathrm{x}$ larger than in a planar reflector arrangement. It is due to this high local field effect that the parabolic reflector nanoantenna results in a stronger Raman signal as compared to the circular waveguide nanoantenna (of Figure 2(c)), which produces only $1.2 x$ enhancement of the local field by the introduction of the circular ring around the dipole over a ground plane.

\section{Raman Scattering Experiments}

Raman scattering experiments were carried out for the fabricated circular waveguide and parabolic antennas using Rhodamine $6 \mathrm{G}$ as the Raman dye excited by a $785 \mathrm{~nm}$ laser. For this CW excitation, there is negligible two-photon fluorescence, which would show up as background in the Raman spectrum. The emitted wavelength at $1509 \mathrm{~cm}^{-1}$ Stokes line $(\lambda=890 \mathrm{~nm})$ was measured using a Renishaw inVia Raman microscope with a $100 \mathrm{x}$ objective and a spot size " $d$ " of approximately $1.5 \mu \mathrm{m}$ (as determined by mapping experiments). Raman dye, Rhodamine $6 \mathrm{G}(400 \mu \mathrm{M}$ in ethanol), was drop-coated, and the sample was allowed to dry for 6 hours.

Figure 7(a) shows the measured Raman signal intensity as a function of Raman shift using the circular waveguide nanoantenna. The circular reflector with radius of $250 \mathrm{~nm}$ resulted in the strongest DERS signal as is predicted by the numerical results of Figure 4.

For comparison, detected intensities from dipole over ground and from the unmilled regions of the top gold surface (without any dipole antenna) are also shown. A dipole antenna of the same dimensions and on the same substrate was fabricated by removing a ring of diameter $10 \mu \mathrm{m}$, to approximate the absence of the ring structure. This dipole over ground is used as the reference for the calculation of EF. The measured spot size of $1.5 \mu \mathrm{m}$ results in illumination of an area of $1.76(\mu \mathrm{m})^{2}$, which is much larger than the area of the antenna gap. From the results of Figure 7(a), it can be seen that the contribution from the gold surface alone (i.e., without the antenna) is small when compared to that from the antenna. It should be noted that the Raman scattering intensity is boosted about $5.5 \mathrm{x}$ as compared to 


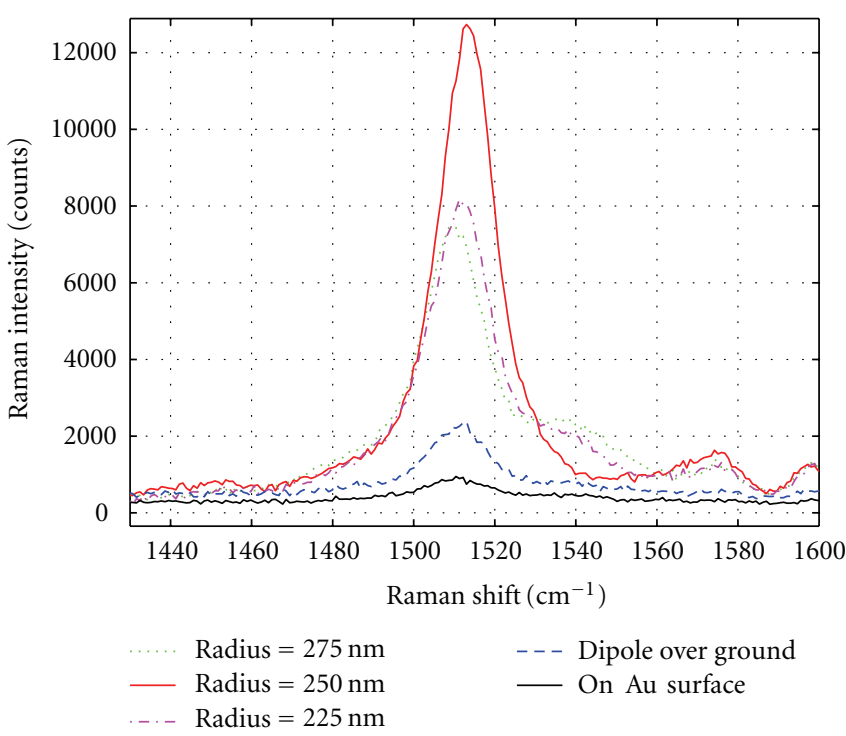

(a)

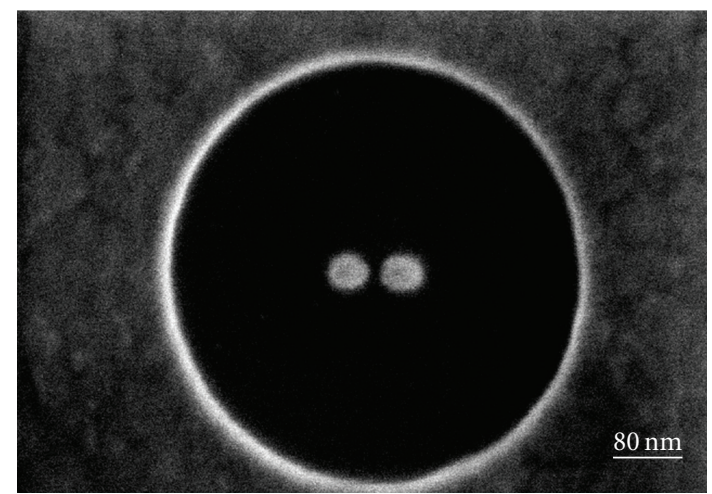

(b)

FIGURE 7: (a) Raman spectra from the nanoantenna structure for various radii of circular reflector, dipole over a ground plane, and the top unmilled Au surface. (b) Scanning electron microscopy image of the fabricated circular waveguide nanoantenna.

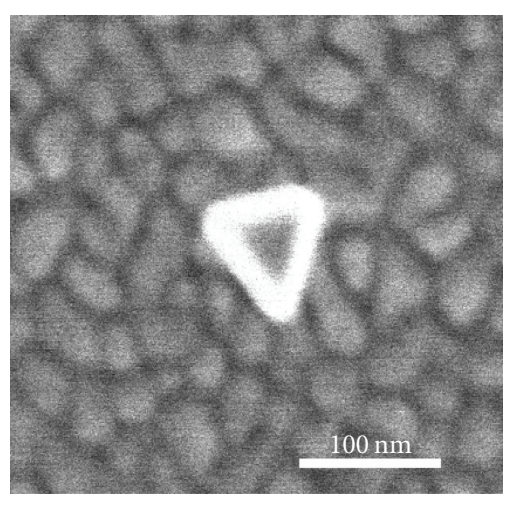

(a)

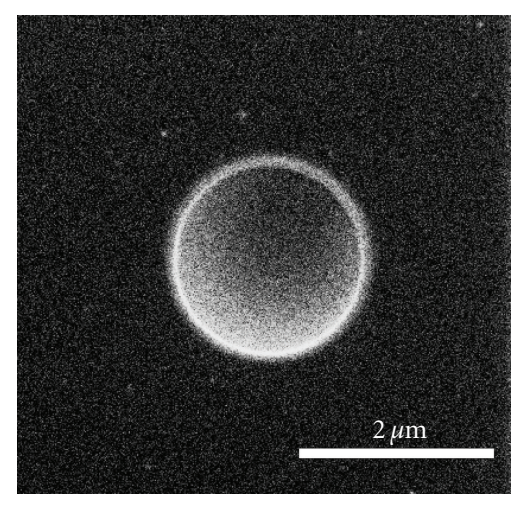

(b)

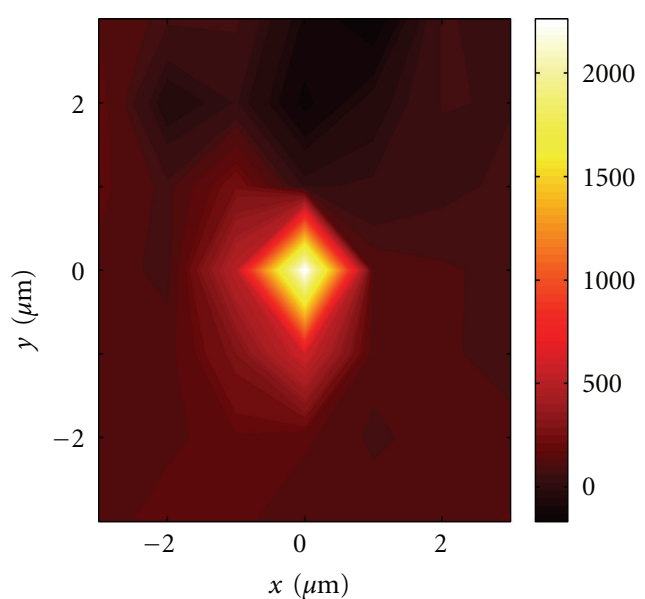

(c)

Figure 8: (a) Scanning electron microscopy image of Ag nanoprism. (b) A scanning ion microscope image of the paraboloid drilled on silicon taken directly from the FIB. (c) A $6 \times 6 \mu \mathrm{m}^{2}$ map of the Raman signal around a parabolic reflector nanoantenna.

dipole over ground and about $13 \mathrm{x}$ with respect to the top Au surface. Note that this $5.5 \mathrm{x}$ increase is in addition to the $50 \mathrm{x}$ signal increase by use of the ground plane [37], and this additional enhancement is attributed to the improved directivity of the ring structure. Thus, the total enhancement from the circular waveguide nanoantenna can be estimated to be $275 \mathrm{x}$ as compared to nanoparticles on a glass substrate. Figure 7(b) shows an SEM image of the fabricated circular waveguide nanoantenna.

To demonstrate that the observed increase in Raman intensity is mainly from antenna directivity and with only a small contribution from local field enhancement, we calculated the enhancement factor arising from local electric field $\left(\mathrm{EF}_{\mathrm{loc}}\right)$ in the antenna feed gap. $\mathrm{EF}_{\mathrm{loc}}=\left|E_{\text {exc }}\right|^{2}\left|E_{\text {scat }}\right|^{2}$, where $\left|E_{\text {exc }}\right|^{2}$ and $\left|E_{\text {scat }}\right|^{2}$ are the electric field intensities at the excitation wavelength of $785 \mathrm{~nm}$ and Stokes shifted scattered radiation at wavelength of $890 \mathrm{~nm}$, respectively. We observe maximum $\mathrm{EF}_{\text {loc }}=1.2$. The measured $5.5 \mathrm{x}$ increase in Raman intensity is thus predominantly from improved directivity of the antenna. Numerically calculated EF using beam efficiency and radiation patterns of Figure 4 with cone angle of 30 degrees is in excellent agreement with the experimental results.

Figure 8(a) shows an SEM of an Ag nanoprism, the scanning ion microscopy image of the fabricated structure (Figure $8(\mathrm{~b})$ ), and a $6 \times 6 \mu \mathrm{m}^{2}$ map of the Raman signal 
using the $1509 \mathrm{~cm}^{-1}$ Stokes line around a parabolic reflector nanoantenna (Figure 8(c)). A clear enhancement to the Raman signal is obtained from the parabolic reflector nanoantenna as compared to the surrounding area, where it is equivalent to a planar reflector nanoantenna. By comparing the maximum Raman signal from a parabolic reflector nanoantenna to the average surrounding signal, a $22 \mathrm{x}$ enhancement is obtained.

Our previous experiments on Ag nanoprism over planar reflector experiments have already shown a 50x Raman enhancement as compared to Ag nanoprisms on glass (without any reflector) [37]. Combining the result of this work and [37], we estimate that our designed parabolic reflector structure can enhance the SERS signal from metallic nanoprisms by $1100 x$. In other words, by directivity engineering, we have boosted the SERS signal from isolated metallic nanoprisms by 3 orders of magnitude.

We confirm that this high signal is not from an aggregation of nanoprisms by taking an SEM image of the antenna. We explain the Raman enhancement with respect to only nanoprisms on glass substrate by the high directivity from the parabolic antenna and enhanced local fields. In an SERS experiment where the nanoprisms are deposited directly onto glass, the light emission is in favor of the direction into the glass substrate due to higher refractive index of the glass. In comparison, the parabolic antenna gives an emission pattern with a tightly focused lobe into the air, with nearly all emitted light directed into the collecting microscope objective. In other words, we have improved the near- and far-field coupling from a poor efficiency to a nearly perfect efficiency by the parabolic antenna. Therefore, the $1100 x$ enhancement between the two cases is not surprising.

The 22x SERS enhancement over the planar reflector from our experiment is very encouraging but is still an underestimate of the parabolic reflector nanoantenna DERS enhancement potential—still lower than our theoretical prediction of 40x enhancement. This may be due to the misalignment between the Ag nanoprism feed element and the Au parabolic reflector in the nanoantenna. The drop-coating technique is convenient to deposit the Ag nanoprisms; however, exactly controlling the nanoprism position is difficult. In future steps, we plan to build the metal feed element using lithographical methods such as FIB lithography. In that way, the position of the feed element can be precisely controlled, and an even higher SERS enhancement is hypothesized.

\section{Conclusions}

We have demonstrated directivity-enhanced Raman scattering (DERS) using directive nanoantennas including parasitic elements and parabolic and waveguide designs. The circular waveguide nanoantenna with a feed gap of $20 \mathrm{~nm}$ produces an enhancement factor of $275 \mathrm{x}$ as compared to nanoparticees over a glass substrate. This enhancement factor can be further increased by reducing the feed gap. Parabolic reflector results in an overall enhancement factor of $1100 \mathrm{x}$ as compared to nanoprisms over a glass substrate; however, as demonstrated in this work, the fabrication of the parabolic structure is more challenging. The enhancement in these waveguide designs is specifically attributed to directivity effects, that is, beam-shaping the antenna cone to fall within the numerical aperture of the imaging optics. Therefore, DERS is separate from other near-field enhancements that arise, for example, from plasmonic effects. Considering the numerical aperture of our microscope Raman system, the nanoantenna presented has near-perfect excitation and collection of the electromagnetic energy. This work is also exciting for related applications, for example, photovoltaics, light-emitting applications, microscopy, sensing, and singlemolecule detection $[29,31,36]$.

\section{Acknowledgment}

The authors acknowledge support from NSERC Strategic Network for Bioplasmonic Systems (BiopSys), Canada.

\section{References}

[1] P. Bharadwaj et al., "Optical antennas," Advances in Optics and Photonics, vol. 1, pp. 438-483, 2009.

[2] H. Yagi, "Beam transmission of ultra short waves," Proceedings of the IEEE, vol. 16, pp. 715-741, 1928.

[3] A. Hartschuh, H. Qian, C. Georgi, M. Böhmler, and L. Novotny, "Tip-enhanced near-field optical microscopy of carbon nanotubes," Analytical and Bioanalytical Chemistry, vol. 394, no. 7, pp. 1787-1795, 2009.

[4] S. Kawata and Y. Inouye, "Scanning probe optical microscopy using a metallic probe tip," Ultramicroscopy, vol. 57, no. 2-3, pp. 313-317, 1995.

[5] C. Fumeaux, M. A. Gritz, I. Codreanu, W. L. Schaich, F. J. González, and G. D. Boreman, "Measurement of the resonant lengths of infrared dipole antennas," Infrared Physics and Technology, vol. 41, no. 5, pp. 271-281, 2000.

[6] F. Neubrech, T. Kolb, R. Lovrincic et al., "Resonances of individual metal nanowires in the infrared," Applied Physics Letters, vol. 89, no. 25, Article ID 253104, 2006.

[7] L. Novotny, "Effective wavelength scaling for optical antennas," Physical Review Letters, vol. 98, no. 26, Article ID 266802, 2007.

[8] S. B. Hasan, R. Filter, A. Ahmed et al., "Relating localized nanoparticle resonances to an associated antenna problem," Physical Review B, vol. 84, no. 19, 2011.

[9] J. N. Farahani, D. W. Pohl, H. J. Eisler, and B. Hecht, "Single quantum dot coupled to a scanning optical antenna: a tunable superemitter," Physical Review Letters, vol. 95, no. 1, Article ID 017402, pp. 1-4, 2005.

[10] S. Kühn, U. Håkanson, L. Rogobete, and V. Sandoghdar, "Enhancement of single-molecule fluorescence using a gold nanoparticle as an optical nanoantenna," Physical Review Letters, vol. 97, no. 1, Article ID 017402, 2006.

[11] T. H. Taminiau, F. D. Stefani, F. B. Segerink, and N. F. Van Hulst, "Optical antennas direct single-molecule emission," Nature Photonics, vol. 2, no. 4, pp. 234-237, 2008.

[12] A. Sundaramurthy, P. J. Schuck, N. R. Conley, D. P. Fromm, G. S. Kino, and W. E. Moerner, "Toward nanometer-scale optical photolithography: utilizing the near-field of bowtie optical nanoantennas," Nano Letters, vol. 6, no. 3, pp. 355-360, 2006.

[13] A. G. Curto, G. Volpe, T. H. Taminiau, M. P. Kreuzer, R. Quidant, and N. F. Van Hulst, "Unidirectional emission of a 
quantum dot coupled to a nanoantenna," Science, vol. 329, no. 5994, pp. 930-933, 2010.

[14] H. Wang, D. W. Brandl, F. Le, P. Nordlander, and N. J. Halas, "Nanorice: a hybrid plasmonic nanostructure," Nano Letters, vol. 6, no. 4, pp. 827-832, 2006.

[15] D. Dregely, R. Taubert, J. Dorfmüller, R. Vogelgesang, K. Kern, and H. Giessen, "3D optical Yagi-Uda nanoantenna array," Nature Communications, vol. 2, no. 1, 2011.

[16] P. Mühlschlegel, H. J. Eisler, O. J. F. Martin, B. Hecht, and D. W. Pohl, "Applied physics: resonant optical antennas," Science, vol. 308, no. 5728, pp. 1607-1609, 2005.

[17] P. J. Schuck, D. P. Fromm, A. Sundaramurthy, G. S. Kino, and W. E. Moerner, "Improving the mismatch between light and nanoscale objects with gold bowtie nanoantennas," Physical Review Letters, vol. 94, no. 1, Article ID 017402, 2005.

[18] P. Anger, P. Bharadwaj, and L. Novotny, "Enhancement and quenching of single-molecule fluorescence," Physical Review Letters, vol. 96, no. 11, Article ID 113002, pp. 1-4, 2006.

[19] T. Kosako, Y. Kadoya, and H. F. Hofmann, "Directional control of light by a nano-optical Yagi-Uda antenna," Nature Photonics, vol. 4, no. 5, pp. 312-315, 2010.

[20] S. Graells, S. Aćimović, G. Volpe, and R. Quidant, "Direct growth of optical antennas using e-beam-induced gold deposition," Plasmonics, vol. 5, no. 2, pp. 135-139, 2010.

[21] E. Ozbay, "Plasmonics: merging photonics and electronics at nanoscale dimensions," Science, vol. 311, no. 5758, pp. 189193, 2006

[22] S. Wedge, J. A. E. Wasey, W. L. Barnes, and I. Sage, "Coupled surface plasmon-polariton mediated photoluminescence from a top-emitting organic light-emitting structure," Applied Physics Letters, vol. 85, no. 2, pp. 182-184, 2004.

[23] R. Corkish, M. A. Green, and T. Puzzer, "Solar energy collection by antennas," Solar Energy, vol. 73, no. 6, pp. 395401, 2002.

[24] S. Pillai, K. R. Catchpole, T. Trupke, and M. A. Green, "Surface plasmon enhanced silicon solar cells," Journal of Applied Physics, vol. 101, no. 9, Article ID 093105, 2007.

[25] H. R. Stuart and D. G. Hall, "Absorption enhancement in silicon-on-insulator waveguides using metal island films," Applied Physics Letters, vol. 69, no. 16, pp. 2327-2329, 1996.

[26] L. Tang, S. E. Kocabas, S. Latif et al., "Nanometre-scale germanium photodetector enhanced by a near-infrared dipole antenna," Nature Photonics, vol. 2, no. 4, pp. 226-229, 2008.

[27] A. M. Marks, "Device for conversion of light to electric power," U.S. Patent 4,445,050, 1984.

[28] C. C. Neacsu, J. Dreyer, N. Behr, and M. B. Raschke, "Scanning-probe Raman spectroscopy with single-molecule sensitivity," Physical Review B, vol. 73, no. 19, Article ID 193406, 2006.

[29] N. Liu, M. L. Tang, M. Hentschel, H. Giessen, and A. P. Alivisatos, "Nanoantenna-enhanced gas sensing in a single tailored nanofocus," Nature Materials, vol. 10, no. 8, pp. 631-636, 2011.

[30] S. A. Maier, "Plasmonic field enhancement and SERS in the effective mode volume picture," Optics Express, vol. 14, no. 5, pp. 1957-1964, 2006.

[31] S. Nie and S. R. Emory, "Probing single molecules and single nanoparticles by surface-enhanced Raman scattering," Science, vol. 275, no. 5303, pp. 1102-1106, 1997.

[32] H. Xu, E. J. Bjerneld, M. Käll, and L. Börjesson, “Spectroscopy of single hemoglobin molecules by surface enhanced Raman scattering," Physical Review Letters, vol. 83, no. 21, pp. 43574360, 1999.
[33] J. Kim, O. Benson, H. Kan, and Y. Yamamoto, "A single-photon turnstile device," Nature, vol. 397, no. 6719, pp. 500-503, 1999.

[34] B. Lounis and W. E. Moerner, "Single photons on demand from a single molecule at room temperature," Nature, vol. 407, no. 6803, pp. 491-493, 2000.

[35] T. Kalkbrenner, U. Håkanson, A. Schädle, S. Burger, C. Henkel, and V. Sandoghdar, "Optical microscopy via spectral modifications of a nanoantenna," Physical Review Letters, vol. 95, no. 20, Article ID 200801, pp. 1-4, 2005.

[36] C. Höppener and L. Novotny, "Antenna-based optical imaging of single $\mathrm{Ca}^{2+}$ transmembrane proteins in liquids," Nano Letters, vol. 8, no. 2, pp. 642-646, 2008.

[37] Q. Min, Y. Pang, D. J. Collins et al., "Substrate-based platform for boosting the surface-enhanced Raman of plasmonic nanoparticles," Optics Express, vol. 19, no. 2, pp. 1648-1655, 2011.

[38] A. Ahmed and R. Gordon, "Directivity enhanced raman spectroscopy using nanoantennas," Nano Letters, vol. 11, no. 4, pp. 1800-1803, 2011.

[39] C. A. Balanis, Antenna Theory Analysis and Design, John Wiley and Sons, NJ, USA, 3rd edition, 2005.

[40] T. Pakizeh and M. Käll, "Unidirectional ultracompact optical nanoantennas," Nano Letters, vol. 9, no. 6, pp. 2343-2349, 2009.

[41] A. Alù and S. Maslovski, "Power relations and a consistent analytical model for receiving wire antennas," IEEE Transactions on Antennas and Propagation, vol. 58, no. 5, Article ID 5422725, pp. 1436-1448, 2010.

[42] A. Al̀ and N. Engheta, "Tuning the scattering response of optical nanoantennas with nanocircuit loads," Nature Photonics, vol. 2, no. 5, pp. 307-310, 2008.

[43] T. J. Seok, A. Jamshidi, M. Kim et al., "Radiation engineering of optical antennas for maximum field enhancement," Nano Letters, vol. 11, no. 7, pp. 2606-2610, 2011.

[44] R. Jin, Y. Cao, C. A. Mirkin, K. L. Kelly, G. C. Schatz, and J. G. Zheng, "Photoinduced conversion of silver nanospheres to nanoprisms," Science, vol. 294, no. 5548, pp. 1901-1903, 2001.

[45] D. M. Pozar, Microwave Engineering, John Wiley and Sons, New York, NY, USA, 1998.

[46] D. R. Ward, N. K. Grady, C. S. Levin et al., "Electromigrated nanoscale gaps for surface-enhanced Raman spectroscopy," Nano Letters, vol. 7, no. 5, pp. 1396-1400, 2007.

[47] J. Theiss, P. Pavaskar, P. M. Echternach, R. E. Muller, and S. B. Cronin, "Plasmonic nanoparticle arrays with nanometer separation for high-performance SERS substrates," Nano Letters, vol. 10, no. 8, pp. 2749-2754, 2010. 

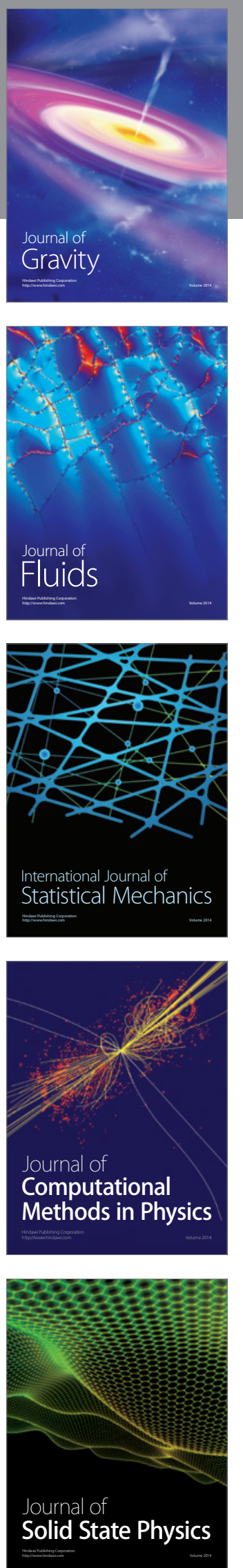

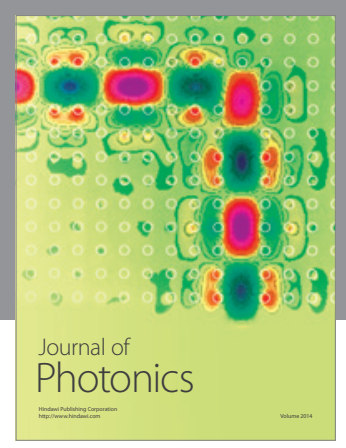

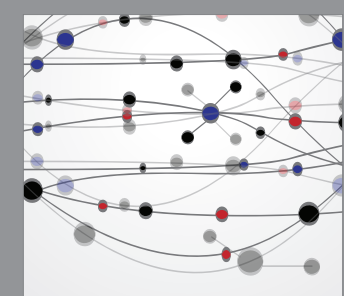

The Scientific World Journal
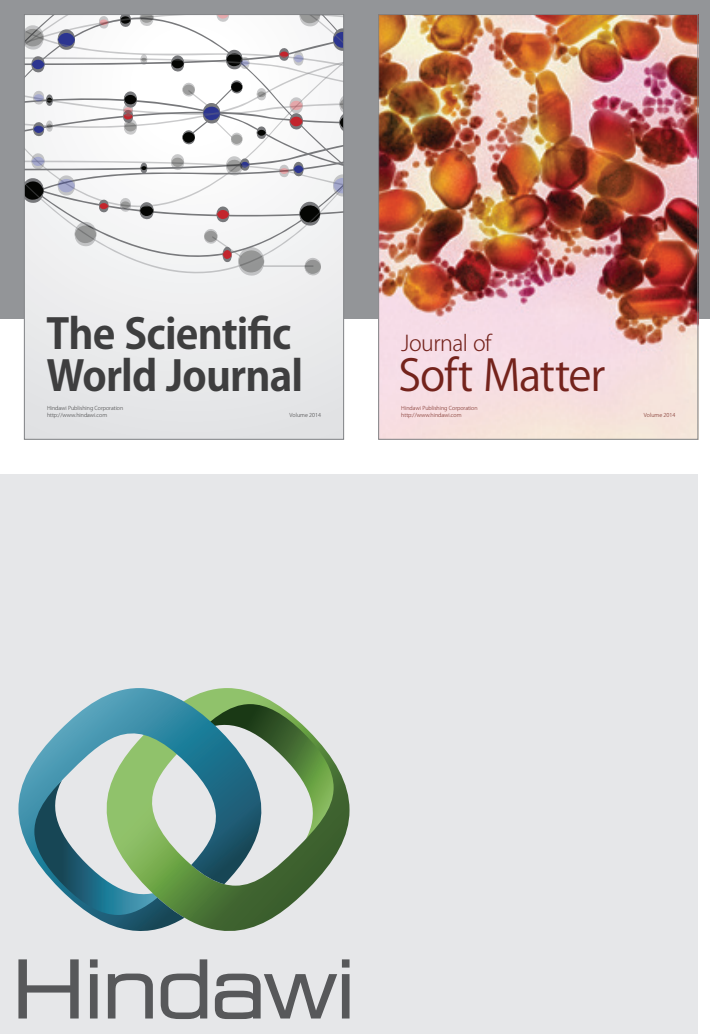

Submit your manuscripts at

http://www.hindawi.com
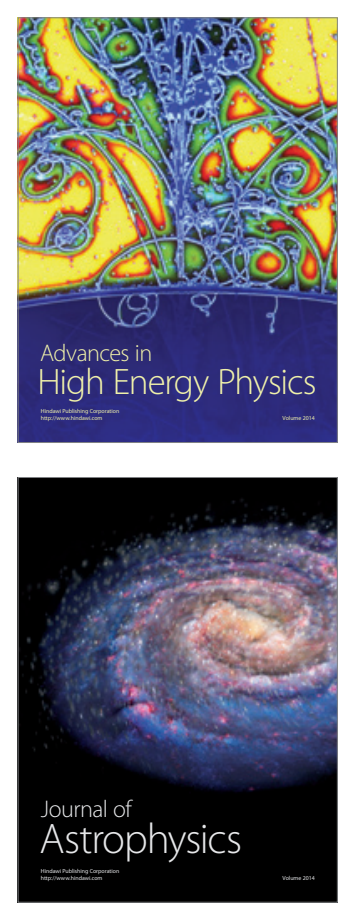
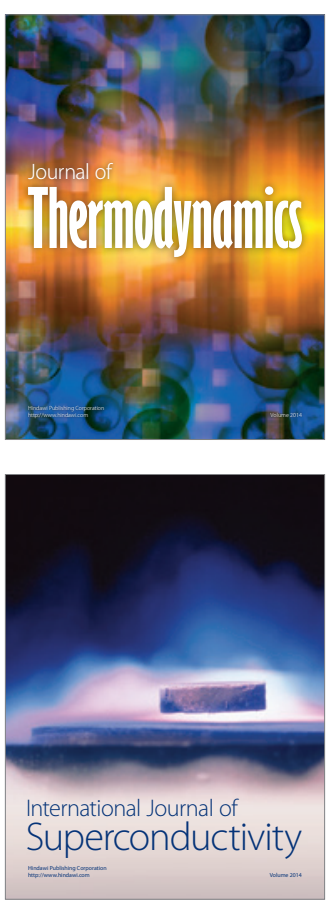
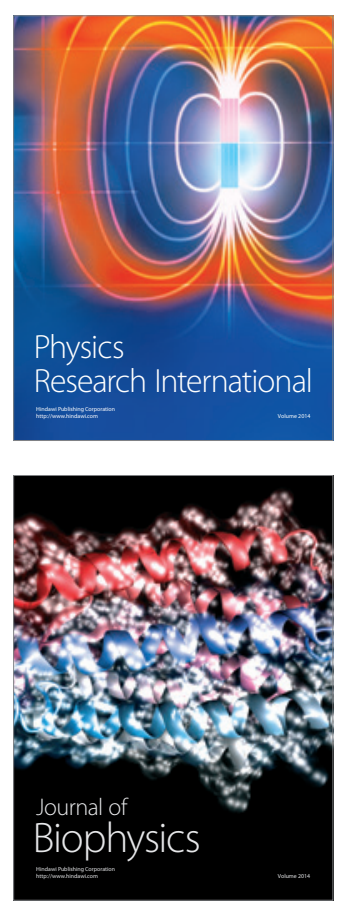
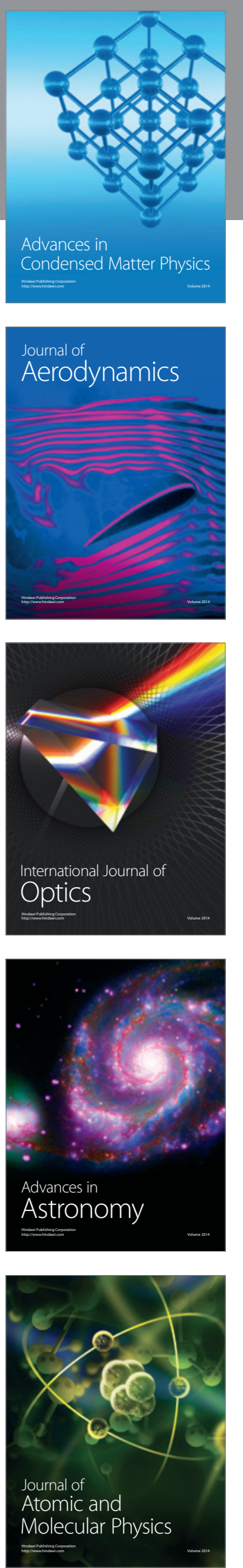